\title{
Incidencia del síndrome de miembro fantasma por amputación de extremidad inferior en el Hospital Santo Tomás de mayo a julio 2019
}

\section{Phantom limb syndrome incidence by amputation of lower limb at Santo Tomas Hospital from may to july 2019}

\author{
Mariel Alanna Armuelles Pinzón (0*; Marjorie Errigo Pinillot৫ \\ *Médico Funcionario Especialista en Anestesiología, Hospital Aquilino Tejeira, Panamá. \\ †Médico Funcionario Especialista en Anestesiología y Algiología, Hospital Santo Tomás, Panamá.
}

\section{RESUMEN}

El síndrome de miembro fantasma está conformado por las siguientes tres entidades: sensación de miembro fantasma, dolor de miembro fantasma y dolor de muñón. La etiología no está claramente establecida y su fisiopatología involucra mecanismos nerviosos periféricos y centrales, además de aspectos psicológicos.

Se llevó a cabo un estudio observacional prospectivo en 28 pacientes. A cada uno se le realizó una evaluación antes, durante y hasta 1 mes después de la cirugía, con el objetivo de determinar la incidencia de este síndrome en los pacientes a los que se realizó amputación de alguna parte del miembro inferior. De los pacientes amputados 23 (82 $\%)$ fueron pacientes con diabetes mellitus. Se obtuvo una incidencia de $17.86 \%$ de síndrome de miembro fantasma. Al mes de realizado el procedimiento quirúrgico $13(46 \%)$ de los casos presentaron sensación de miembro fantasma, 8 (27\%) dolor de miembro fantasma y 7 ( $24 \%$ ) dolor de muñón.

\section{ABSTRACT}

Phantom limb syndrome is made up of the following three entities: phantom limb sensation, phantom limb pain and stump pain. The etiology is not clearly established and its pathophysiology involves peripheral, central and psychological mechanisms.

A prospective observational study was carried out in 28 patients, an evaluation was made on the patient before, and up to 1 month after surgery, with the aim of determining the incidence of this syndrome in patients who underwent amputation of any part of the lower limb. Twenty-three (82\%) of amputees were patients with diabetes mellitus and an incidence of $17.86 \%$ of phantom limb syndrome was obtained. One month after the surgical procedure, $8(27 \%)$ of the cases presented phantom limb pain, $7(24 \%)$ stump pain and 13 (46\%) phantom limb sensation.

\section{Correspondencia:} marielita0589@gmail.com Recibido: 24/03/2020 Aceptado: 21/04/2021

\section{PALABRAS CLAVE:}

Miembro fantasma; dolor en el miembro fantasma; seudomelia KEY WORDS:

Phantom limb; phantom limb pain; pseudomelia

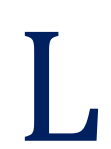

a primera ocasión en que se realizó la descripción de sensación de miembro fantasma originada posterior a una amputación, fue en el año 1552 por el cirujano francés Ambroise Paré. En el año 1872, se introduce el término "dolor de miembro fantasma" por el neurólogo norteamericano Silas Weir Mitchel. ${ }^{[1]}$

El síndrome de miembro fantasma (SMF) está compuesto por tres entidades que coexisten frecuentemente:

- Sensación de miembro fantasma: cualquier sensación en ausencia de un miembro, excepto dolor. ${ }^{[2]}$ 
- Dolor de miembro fantasma (DMF): es la sensación dolorosa que ocurre después de una amputación, referida hacia la parte distal de la región en que se encontraba previamente la extremidad. ${ }^{[2]}$

o Dolor del muñón (DM): dolor localizado en el muñón de la extremidad amputada. ${ }^{[2]}$

El dolor de miembro fantasma es un tipo de dolor neuropático. Este se define como el dolor que se presenta como consecuencia directa de una lesión o enfermedad que afecta al sistema somatosensorial periférico o central según la Asociación Internacional para el Estudio del Dolor. ${ }^{[3]}$

Actualmente no existe consenso acerca de la prevalencia del síndrome de miembro fantasma. Se estima que puede oscilar entre $49 \%$ y $83 \%$ de los pacientes amputados ${ }^{[4]}$ y la incidencia puede variar desde $2 \%$ al $80 \%$, lo cual se atribuye a que muchos pacientes no reportan el dolor por temor a ser estigmatizados. Estudios prospectivos demuestran que el mismo se presenta durante las primeras 24 horas postamputación hasta en el $50 \%$ de los casos. ${ }^{[5]}$

En los países de Occidente, las principales causas de amputación son diabetes mellitus, traumatismo, enfermedad vascular crónica y tumores. ${ }^{[4]}$

La etiología del síndrome de miembro fantasma no está claramente establecida. Los estudios revelan tres hipótesis acerca de su origen y mecanismo: periférico, medular y central (supraespinal). ${ }^{[6]}$

Clínicamente se puede presentar con una variedad de sensaciones como cambios de temperatura, prurito, parestesia, sensaciones de impulsos eléctricos o percepción del miembro, el cual puede seguir ordenes o estar paralizado, doblado o torcido.
Las sensaciones dolorosas incluyen ardor, sensación pulsante o punzante, las cuales pueden ser espasmódicas o persistentes. La calidad del dolor puede cambiar con el tiempo y presentarse de forma transitoria. ${ }^{[7,8]}$

En cuanto a la prevención hay evidencia de que la utilización de analgesia perioperatoria rigurosa reduce la prevalencia del DMF. ${ }^{[9,10]}$

Con respecto al tratamiento no hay un consenso establecido; pero, se sabe que un tratamiento temprano mejora el pronóstico y las posibilidades de éxito terapéuticas. El manejo descrito incluye medidas farmacológicas y no farmacológicas. ${ }^{[11,12]}$

Esta investigación se llevó a cabo sin conflicto de intereses y tiene el objetivo de determinar la incidencia con la que se presentó el síndrome de miembro fantasma en los pacientes con amputación de miembro inferior en el Hospital Santo Tomás de mayo a julio 2019. Además de señalar las características demográficas, determinar los principales diagnósticos asociados, describir si los pacientes presentan algún tipo de dolor, su intensidad y manejo en el preoperatorio y en el postoperatorio, indicar el abordaje anestésico para las amputaciones de miembro inferior, conocer si los pacientes presentan dolor de miembro fantasma, dolor en el muñón y sensaciones de miembro fantasma hasta un mes de realizado el procedimiento quirúrgico.

\section{MATERIALES Y MÉTODOS}

Este estudio prospectivo observacional descriptivo se llevó a cabo en el periodo de mayo a julio del año 2019 en todos los pacientes a los que se le amputó alguna parte del miembro inferior en el Hospital Santo Tomás, luego de la aprobación por el comité 
de ética e investigación del Hospital Dr. José Renán Esquivel.

\section{Tamaño de la Muestra}

$n=N Z^{2} p(1-p) /(N-1) e^{2}+Z^{2} p(1-p)$

$\mathrm{N}$ : todos los pacientes a los que se le realizó amputación de miembro inferior en el año $2018=$ 144 pacientes

$\mathrm{Z}=1.96 \mathrm{P}=0.5 \mathrm{e}=5 \%$ para un nivel de confianza de $95 \% \mathrm{n}=104$ para el año 2019

$\mathrm{n}=26$ pacientes en 3 meses finalmente se incluyeron 28 pacientes al estudio.

Tomando en cuenta que en el año 2018 se realizaron 144 amputaciones de miembro inferior, se obtuvo que el tamaño de la muestra fue de 26 pacientes para un periodo de estudio de 3 meses. Participaron en el estudio los pacientes a los que se les realizó una amputación de miembro inferior ya sea por urgencia o de forma electiva y que cumplían con los criterios de inclusión. A todos se les evaluó en el preoperatorio la presencia de dolor en la extremidad antes de la amputación, si recibía algún tratamiento, si usaba ansiolíticos y sus diagnósticos preoperatorios. Luego se tomaron los siguientes datos de la hoja de anestesia: nivel de amputación, servicio que realizó la amputación, tipo de anestesia utilizada, analgesia administrada en el transoperatorio. En el postoperatorio se evaluó la presencia de sensación de miembro fantasma, presencia e intensidad de dolor de miembro fantasma mediante la escala verbal numérica y dolor en el muñón a las 24 horas, a la semana y al mes de realizado el procedimiento quirúrgico. Luego se creó una base de datos en el programa estadístico Epi info versión 7.2.2.6 y en Excel, donde se realizó el análisis de los resultados.
Se establecieron como criterios de inclusión:

- Edad mayor de 18 años.

- Amputación unilateral de extremidad inferior (por encima de la rodilla, por debajo de la rodilla, transmetatarsiana y dedos de los pies).

- Consentimiento informado del paciente para participar en el estudio.

Y como criterios de exclusión:

- Clasificación perioperatoria según el estado físico de la Sociedad Americana de Anestesiología (ASA) IV.

- Negación del paciente a participar en el estudio.

RESULTADOS

Evaluamos 28 pacientes a los que se les realizó amputación de alguna parte del miembro inferior durante el periodo comprendido entre mayo y julio 2019. Según los datos obtenidos 18 (64\%) de los casos pertenecen al sexo masculino. También se observó que 15 (54 \%) pacientes son mayores de 60 años, 11 (39 \%) pacientes se encuentran en el rango etario de 30 a 60 años y sólo 2 (7\%) pacientes eran menores de 30 años. La mayoría de los pacientes amputados padecían de diabetes mellitus (Ver Gráfica 1).

A ningún paciente se le administró ansiolíticos en el preoperatorio y a 18 (64\%) de los casos, se les colocó algún analgésico antes de la cirugía. Entre las combinaciones más utilizadas se encuentran antinflamatorios no esteroideos (AINES) y opioides débiles, específicamente tramadol (Ver Gráfica 2). 


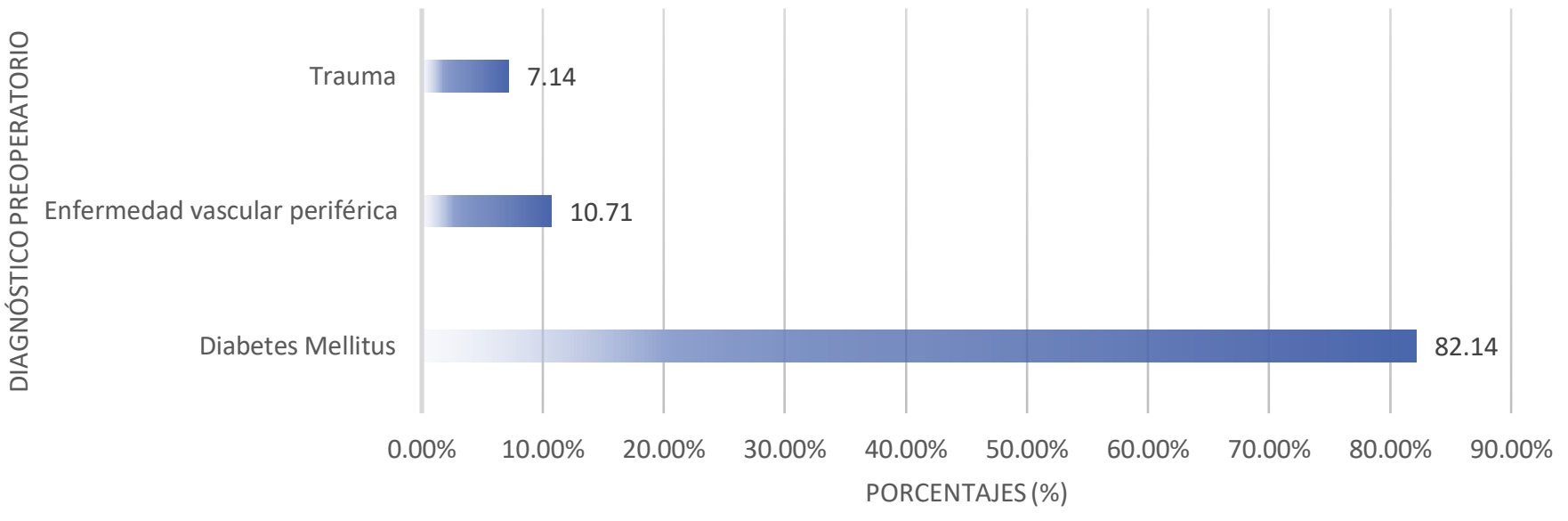

Gráfica 1. Diagnóstico preoperatorio en pacientes a los que se les realizó amputación de extremidad inferior en el Hospital Santo Tomás de mayo a julio 2019.

Fuente: Hoja de recolección de datos de "Incidencia del síndrome de miembro fantasma por amputación de extremidad inferior en el Hospital Santo Tomás de mayo a julio 2019".

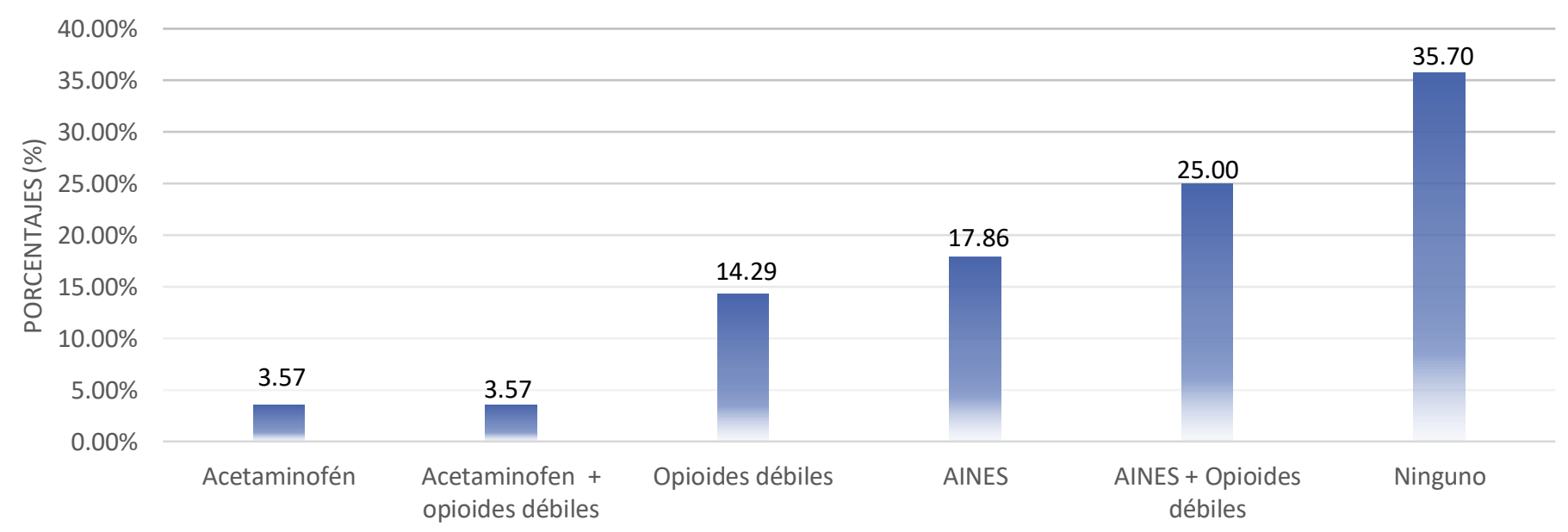

ANALGÉSICOS EN EL PREOPERATORIO

Gráfica 2. Tipos de analgésicos administrados en el preoperatorio de pacientes a los que se les realizó amputación de extremidad inferior en el Hospital Santo Tomás de mayo a julio 2019.

Fuente: Hoja de recolección de datos de "Incidencia del síndrome de miembro fantasma por amputación de extremidad inferior en el Hospital Santo Tomás de mayo a julio 2019".

De los 28 pacientes evaluados, 15 (54\%) de ellos fueron clasificados como ASA III. Además, según los datos se realizaron 18 (64\%) amputaciones por encima de la rodilla, seguido de 9 (32\%) amputaciones de artejos y 1 (3.57\%) amputación transmetatarsiana.
La modalidad anestésica más utilizada para la cirugía fue neuroaxial raquídea, seguido de anestesia general y bloqueo de nervio periférico con el mismo porcentaje. Es importante destacar que el bloqueo de nervio femoral fue el más utilizado (Ver Gráfica 3). 


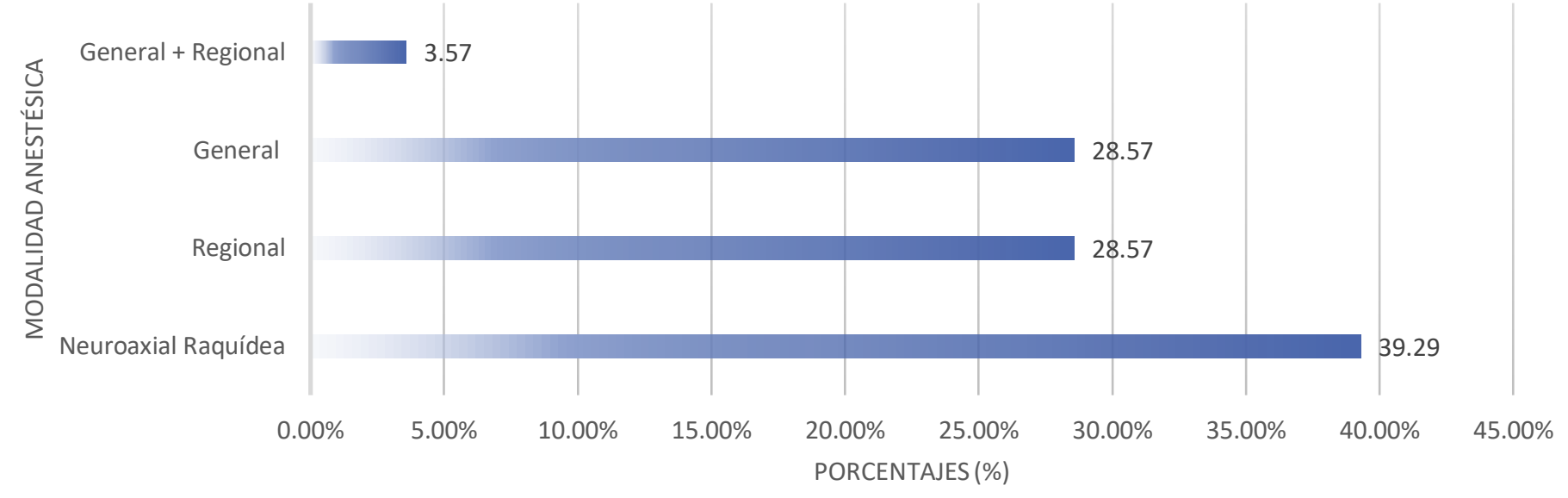

Gráfica 3. Modalidad anestésica empleada para amputación de extremidad inferior en el Hospital Santo Tomás de mayo a julio 2019.

Fuente: Hoja de recolección de datos de Incidencia del síndrome de miembro fantasma por amputación de extremidad inferior en el Hospital Santo Tomás de mayo a julio 2019".

Los AINES fueron los analgésicos más utilizados en el intraoperatorio en 12 de los 28 casos con un 42\%, seguido de la combinación de AINES más opioides débiles representado por un $32 \%$. En cuanto a la analgesia en el postoperatorio, la combinación de AINES y opioides débiles fue la más utilizada (Ver Gráfica 4).
En cuanto al DMF a las 24 horas de la cirugía, lo presentaron 6 de los 28 pacientes: 5 (17\%) moderado y 1 (3.57\%) severo. Una semana después de la cirugía hubo un aumento a 9 pacientes y finalmente, al ser evaluados un mes después del procedimiento quirúrgico, 8 pacientes seguían presentando DMF: 3 (10\%) leve y 5 (17\%) moderado (Ver Gráfica 5).

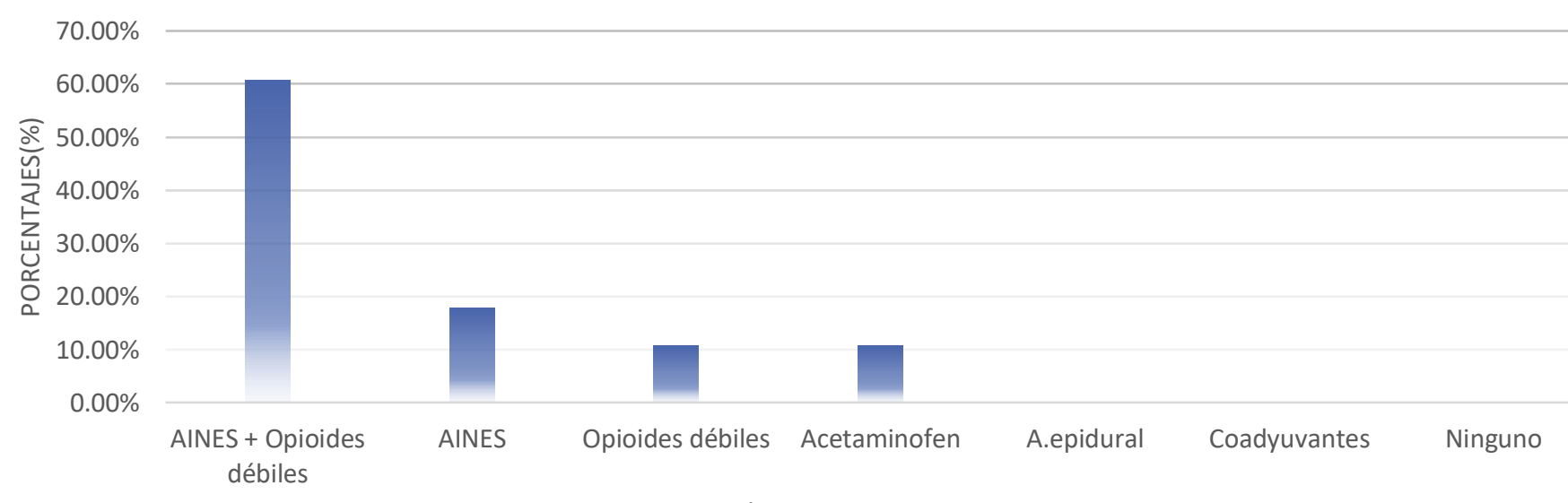

ANALGÉSICOS EN EL POSTOPERATORIO

Gráfica 4. Tipos de analgésicos administrados en el postoperatorio de pacientes a los que se les realizó amputación de extremidad inferior en el Hospital Santo Tomás de mayo a julio 2019.

Fuente: Hoja de recolección de datos de "Incidencia del síndrome de miembro fantasma por amputación de extremidad inferior en el Hospital Santo Tomás de mayo a julio 2019". 


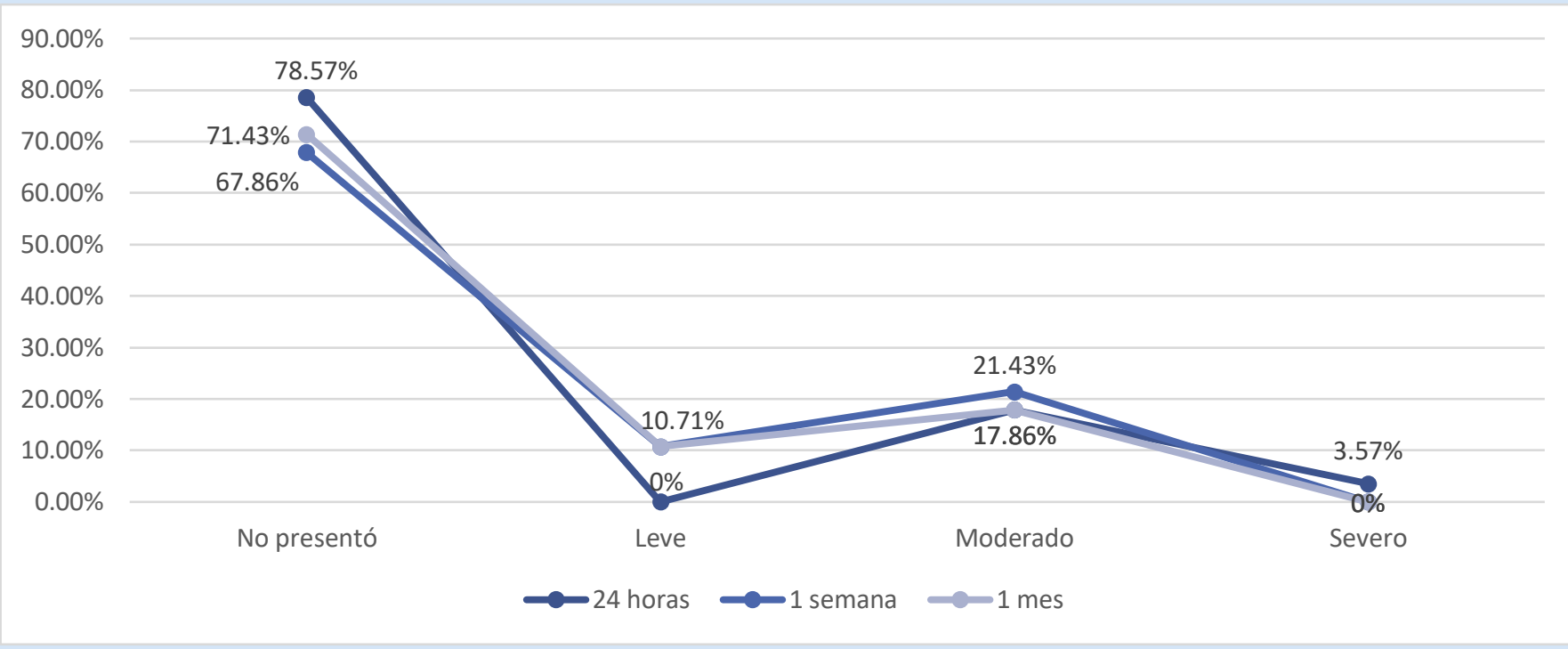

Gráfica 5. Dolor de miembro fantasma por amputación de extremidad inferior en el Hospital Santo Tomás de mayo a julio 2019.

Fuente: Hoja de recolección de datos de Incidencia del síndrome de miembro fantasma por amputación de extremidad inferior en el Hospital Santo Tomás de mayo a julio 2019".

La mayoría de los pacientes 16 (57.1\%) referían dolor de muñón a las 24 horas de la amputación; sin embargo, en las evaluaciones posteriores a la semana y al mes de la cirugía esta cantidad de pacientes disminuyó a 12 (42.9\%) y luego 7 (24.9\%), respectivamente (Ver Tabla 1).

Al mes de realizada la cirugía $13(46.43 \%)$ de los 28 pacientes presentaban sensación de miembro fantasma y el prurito era la sensación que más referían (Ver Tabla 2).

Un total de 5 pacientes presentaron las 3 entidades del SMF, manteniéndose durante todo el periodo de la investigación, con evaluaciones a las 24 horas, a la semana y al mes de la cirugía, lo que representa una incidencia de $17.86 \%$ de este síndrome. De los 5 pacientes que desarrollaron SMF, 4 (80\%) presentaron dolor en la evaluación preoperatoria (Ver Tabla 3).

En cuanto a la modalidad anestésica empleada, de los 5 pacientes que desarrollaron SMF a 3(60\%) se les administró anestesia general para la amputación (Ver Gráfica 6).

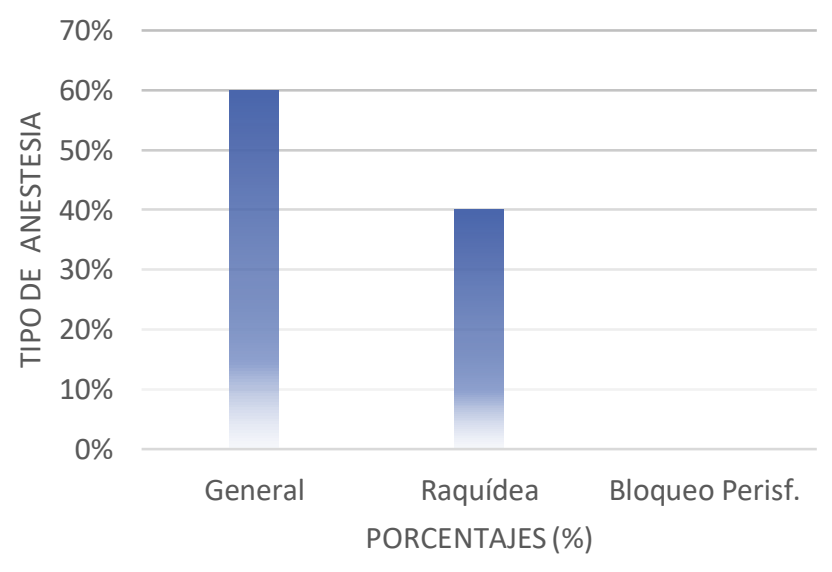

Gráfica 6. Tipo de anestesia administrada en pacientes que desarrollaron síndrome de miembro fantasma por amputación de extremidad inferior en el Hospital Santo Tomás de mayo a julio 2019.

Fuente: Hoja de recolección de datos de "Incidencia del síndrome de miembro fantasma por amputación de extremidad inferior en el Hospital Santo Tomás de mayo a julio 2019". 


\begin{tabular}{|c|c|c|c|c|c|c|}
\hline Dolor de Muñón & 24 horas & Porcentaje & 1 semana & Porcentaje & 1 mes & Porcentaje \\
\hline No presentó & 12 & $42.9 \%$ & 16 & $57.14 \%$ & 21 & $75 \%$ \\
\hline Leve & 1 & $3.57 \%$ & 5 & $17.86 \%$ & 2 & $7.14 \%$ \\
\hline Moderado & 13 & $46.43 \%$ & 7 & $25 \%$ & 5 & $17.85 \%$ \\
\hline Severo & 2 & $7.14 \%$ & 0 & $0 \%$ & 0 & $0 \%$ \\
\hline Total & 28 & $100 \%$ & 28 & $100 \%$ & 28 & $100 \%$ \\
\hline
\end{tabular}

Fuente: Hoja de recolección de datos de: "Incidencia del síndrome de miembro fantasma por amputación de extremidad inferior en el Hospital Santo Tomás de mayo a julio 2019".

\begin{tabular}{|ccccccc|}
\hline $\begin{array}{l}\text { Tabla 2: } \\
\text { inferior en el hospital Santo Tomás de mayo a julio 2019. }\end{array}$ & \multicolumn{7}{l}{. } \\
\hline Sensación MF & 24 horas & Porcentaje & 1 semana & Porcentaje & 1 mes & Porcentaje \\
No presentó & 18 & $64.29 \%$ & 16 & $57.14 \%$ & 15 & $53.57 \%$ \\
Prurito & 4 & $14.29 \%$ & 6 & $21.43 \%$ & 7 & $25.00 \%$ \\
Hormigueo & 5 & $17.86 \%$ & 5 & $17.86 \%$ & 5 & $17.86 \%$ \\
Cambio de posición & 1 & $3.57 \%$ & 1 & $3.57 \%$ & 1 & $3.57 \%$ \\
Total & 28 & $100 \%$ & 28 & $100 \%$ & 28 & $100 \%$ \\
\hline
\end{tabular}

Fuente: Hoja de recolección de datos de "Incidencia del síndrome de miembro fantasma por amputación de extremidad inferior en el Hospital Santo Tomás de mayo a julio 2019".

\begin{tabular}{|c|c|c|}
\hline $\begin{array}{l}\text { Dolor preoperatorio } \\
\text { en pacientes con SMF }\end{array}$ & Frecuencia & Porcentaje \\
\hline Sí & 4 & $80 \%$ \\
\hline No & 1 & $20 \%$ \\
\hline Total & 5 & $100 \%$ \\
\hline
\end{tabular}

Fuente: Hoja de recolección de datos de: Incidencia del síndrome de miembro fantasma por amputación de extremidad inferior en el Hospital Santo Tomás de mayo a julio 2019".

\section{DISCUSIÓN}

Con nuestro estudio buscamos describir las principales características de los pacientes a los cuales se les realiza amputación de miembro inferior y la incidencia del síndrome de miembro fantasma en el Hospital Santo Tomás en el periodo de mayo a julio 2019.
Leiva et al. realizaron un estudio descriptivo, transversal para evaluar incidencia de sensación de miembro fantasma en Honduras en el año 2017, obteniendo en cuanto a la distribución de variables sociodemográficas un predominio de sexo masculino de $75 \%$ y más de $50 \%$ de pacientes con edad mayor a 45 años. Nuestros resultados siguen una distribución similar, con $64 \%$ de los pacientes estudiados de sexo masculino y más del $50 \%$ mayores de 60 años. ${ }^{[13]}$ Por otro lado, Leiva y sus colaboradores señalan como las causas de amputación más frecuentes los accidentes viales con un $27 \%$, seguido de las enfermedades periféricas vasculares con un $24.3 \%$ y el pie diabético $21.6 \%$; mientras que en nuestra investigación las causas que lideraban fueron el pie diabético con $82 \%$, seguido de enfermedad vascular periférica $11 \%$ y trauma con $7 \%{ }^{[13]}$

En 2015 Padovani et al. realizan un estudio descriptivo de cohorte retrospectivo para evaluar las características de los pacientes con dolor de 
miembro fantasma en Brasil, obteniendo que en un $66 \%$ de los casos los pacientes desarrollaron síntomas de ansiedad y en un $40 \%$ depresión. En la evaluación preoperatoria de los pacientes de nuestro estudio ninguno fue manejado con ansiolíticos antes de la cirugía. ${ }^{[14]}$

Tonon et al. realizaron un estudio en 52 pacientes amputados en Brasil en el 2012 para valorar el síndrome de miembro fantasma obteniendo que $42 \%$ de las amputaciones realizadas fueron por encima de la rodilla. En los resultados obtenidos en nuestro estudio las amputaciones por encima de la rodilla representan un $64 \% .{ }^{[15]}$

Schley et al. realizaron un estudio descriptivo retrospectivo para evaluar el miembro fantasma doloroso, no doloroso y dolor de muñón en 96 pacientes con amputación traumática obteniendo que la primera aparición se dio en las primeras horas del procedimiento quirúrgico en los siguientes porcentajes DMF 28\%, Sensación de miembro fantasma $14 \%$ y DM $20 \%$. En comparación con los resultados obtenidos en nuestro estudio, en las primeras 24 horas los porcentajes de cada entidad fueron los siguientes: DMF 21\%, Sensación de miembro fantasma $35 \%$ y DM 57\%. ${ }^{[16]}$

\section{CONCLUSIÓN}

- En la población estudiada se evidenció un predominio de sexo masculino con edades por encima de los 60 años y con diabetes mellitus como principal comorbilidad asociada.

- A la mayoría de los casos se les administró analgesia en el preoperatorio y la combinación más comúnmente utilizada consiste en AINES más opioides débiles.
- La mayoría de los pacientes sometidos a amputación de miembro inferior fueron clasificados como ASA III y el nivel de amputación más comúnmente realizado fue por encima de la rodilla.

- Para el procedimiento quirúrgico la modalidad anestésica principalmente utilizada fue neuroaxial raquídea y la analgesia más utilizada en el intraoperatorio consiste en AINES.

- La mayor presentación de DMF se observó a la semana de haber realizado la cirugía y en la mayoría de los casos el DMF era de intensidad moderada.

- En la mayoría de los casos los pacientes presentaron dolor de muñón a las 24 horas de la cirugía, lo cual fue disminuyendo en frecuencia e intensidad en las evaluaciones correspondientes a la semana y al mes.

- Principalmente la sensación de miembro fantasma se presentó al mes del procedimiento quirúrgico y la sensación más comúnmente descrita por el paciente fue prurito.

- A la mayoría de los pacientes se les administra analgesia en el postoperatorio y la combinación más comúnmente utilizada consiste en AINES más opioides débiles.

- La incidencia de síndrome de miembro fantasma obtenida con los resultados de esta investigación fue de $17.86 \%$. 


\section{BIBLIOGRAFÍA}

[1] Vaquerizo A. Dolor postamputación. Rev. Soc. Esp. del Dolor.2000;7 (2): 60-77.

[2] Criollo-Muñoz FH y Hernández-Santos JR. Dolor de miembro fantasma. Rev Esp Méd Quir. 2016 jul;21(3):100-108.

[3] Vidal J. Manual de medicina del dolor. Sociedad española de Dolor. Editorial médica Panamericana: Editorial;2016.

[4] Velasco A, Arcia D, San martin P, Fresia Solis F. Dolor fantasma en niños y jóvenes amputados adquiridos: prevalencia y características clínicas. Rehabil. Integral. 2015; 10 (1): 8-16.

[5] Malavera M, Carrillo S, Gómez O, García R, Silva F. Fisiopatología y tratamiento del dolor de miembro fantasma. Rev Colomb Anestesiol. 2014; 42:40-6.

[6] Flor $H$, Birbaumer N, Sherman R. Dolor de miembro fantasma. Rev. Soc. Esp. Dolor. 2001;8: 327-331.

[7] Treviño M, Salazar S, Escamilla C, Daniel S, Martínez H, Rivera G. Síndrome del miembro fantasma, dolor real. revista médica MD 2012; 4 (1).

[8] Cuartero D, García A, Diago A, García A. Síndrome de miembro fantasma. Med Gen y Fam. 2012;1(2):85-88.

[9] Rathmell J. P. Kehlet. H. Do We Have the Tools to Prevent Phantom Limb Pain? Anesthesiology 2011; 114:1021-4.

[10] Karanikolas M, Aretha D, Tsolakis L, Monantera G, Kiekkas P, Papadoulas P, et al. La analgesia perioperatoria optimizada reduce la intensidad, prevalencia y frecuencia del dolor crónico de miembro fantasma. Anesthesiology 2011; 114:1144-54.

[11] Alviar M, Hale T, Dungca M. Intervenciones farmacológicas para tratar el dolor de miembro fantasma. Cochrane Database of Systematic Reviews 2016.

[12] Mas Esquerdo J., Maruenda Fernández. R., Robles Sánchez J. Tratamiento neuropsicológico de «dolor de miembro fantasma» a propósito de un caso. Sanid. mil. 2013; 69 (3): 195-202.

[13] Leiva F, Aguilar L, Carcamo H. Sensación del Miembro Fantasma y Dolor del Miembro Residual en la Comunidad. Rev. Med. Gal. Sci. 2017;1.

[14] Padovani $M$, Martins $M$, Venancio $A$, Nogueira J. Ansiedad, depresión y calidad de vida en individuos con dolor de miembro fantasma. Acta Ortop Bras.2015;23(2):107-10.

[15] Tonon da Luz S, Souza J, Andrade M, Ventoza C, Honório G, Avila A, Berral FJ. Valoración del síndrome del dolor fantasma en amputados: abordaje bio-psico-social. Trauma Fund MAPFRE. 2012; 23 (3):176-182.

[16] Schley M, Wilms P, Toepfner S, Schaller H, Schmelz M, Konrad C, Birbaumer N. Sensaciones fantasmas dolorosas, no dolorosas y de muñón en amputaciones traumáticas agudas. J Trauma. 2008; 65:858-864. 